\title{
Repurposing Therapeutics for COVID-19: Supercomputer-Based Docking to the SARS-CoV-2 Viral Spike Protein and Viral Spike Protein-Human ACE2 Interface
}

\author{
Micholas Dean Smith ${ }^{1,2}$ and Jeremy C. Smith ${ }^{1,2,3^{*}}$ \\ * Corresponding author: smithjc@,ornl.gov \\ ${ }^{1}$ Center for Molecular Biophysics, University of Tennessee/Oak Ridge National Laboratory, Oak Ridge National \\ Laboratory, P.O. Box 2008, Oak Ridge, TN 37831 \\ ${ }^{2}$ Department of Biochemistry and Cellular and Molecular Biology, University of Tennessee, M407 Walters Life \\ Sciences, 1414 Cumberland Avenue, Knoxville, TN 37996 \\ ${ }^{3}$ Oak Ridge National Laboratory, P.O. Box 2008, Oak Ridge, TN 37831
}

This manuscript has been authored by UT-Battelle, LLC under Contract No. DE-AC05-00OR22725 with the U.S. Department of Energy. The United States Government retains and the publisher, by accepting the article for publication, acknowledges that the United States Government retains a non-exclusive, paid-up, irrevocable, world-wide license to publish or reproduce the published form of this manuscript, or allow others to do so, for United States Government purposes. The Department of Energy will provide public access to these results of federally sponsored research in accordance with the DOE Public Access Plan (http://energy.gov/downloads/doe-public-access-plan ). 


\begin{abstract}
The novel Wuhan coronavirus (SARS-CoV-2) has been sequenced, and the virus shares substantial similarity with SARS-CoV. Here, using a computational model of the spike protein (S-protein) of SARS-CoV-2 interacting with the human ACE2 receptor, we make use of the world's most powerful supercomputer, SUMMIT, to enact an ensemble docking virtual highthroughput screening campaign and identify small-molecules which bind to either the isolated Viral S-protein at its host receptor region or to the S protein-human ACE2 interface. We hypothesize the identified small-molecules may be repurposed to limit viral recognition of host cells and/or disrupt host-virus interactions. A ranked list of compounds is given that can be tested experimentally.
\end{abstract}

\title{
Introduction
}

On the penultimate day of 2019, health officials at the Wuhan Municipal Health Commission (Hubei Province, China) reported an occurrence of concentrated pneumonia in the city of Wuhan ${ }^{1-2}$. Shortly after reporting the outbreak, the Chinese Center for Disease Control (Chinese CDC) and local Chinese health workers determined that the cause of the outbreak was a novel coronavirus denoted, initially, as the Wuhan Coronavirus or nCov-2019 (and now referred to as SARS-CoV-2) ${ }^{3}$. By Jan. $10^{\text {th }}, 2020$, the genome sequence of nCoV-2019 was released ${ }^{4}$. On Jan. $20^{\text {th }}, 2020, \mathrm{Xu}$ et $\mathrm{al}^{2}$, compared the available sequences of those of other coronaviruses and demonstrated that the novel coronavirus was within the SARS family (SARS-CoV).

Human infections by the SARS coronavirus are known to be closely associated with interactions between the viral spike protein (S-protein) and specific human host receptors, such as the Angiotensin-converting enzyme 2 (ACE2) receptor ${ }^{5-6}$. Using early SARS-CoV-2 genome 
data and standard template-based modeling techniques, $\mathrm{Xu}$ et al. constructed a 3D structural model of the SARS-CoV-2 S-protein. Further, after obtaining the structural model, proteinprotein docking calculations were performed indicating that although SARS-CoV and SARSCoV-2 do have a several differences in their sequences, both have favorable binding affinity for the human ACE2 receptor ${ }^{2}$. Following this initial report, additional work has also provided evidence of the COVID-19 S-protein binding to the ACE2 receptor ${ }^{7-9}$.

The current model of the SARS-CoV-2 S-protein:human ACE2 complex facilitated infection suggests that a reasonable target for structure-based drug discovery might be to disrupt the viral S-protein-ACE2 interface. However, the development of novel small-molecule therapeutics (drugs) takes years, with trials and regulatory approval taking between 10 to 15 years in the US (on average) ${ }^{10}$. Given that the outbreak of SARS-CoV-2may quickly become a global challenge ${ }^{11}$, it would be of great benefit to identify and repurpose already wellcharacterized small-molecules and approved drugs for use in combating the disease.

Here we combine restrained temperature replica-exchange molecular dynamics (restrained T-REMD) simulations with virtual high-throughput screening in an ensemble docking campaign to identify well-characterized drugs, metabolites, and/or natural products that may disrupt S-protein:ACE2 receptor interface stability or the ability of the S-protein to recognize the ACE2 receptor. From this ensemble docking campaign, we provide a ranking of the predicted binding affinities of over 8000 drugs, metabolites, and natural products (and their isomers) with regards to the COVID-19 S-protein and the S-protein:ACE2 receptor. Further, we highlight seven of our top ranked compounds, which are currently available and have had either regulatory approval as drugs or have had multiple prior studies which indicating high-potential for therapeutic use. 


\section{Methods}

Ensemble docking is a strategy by which an ensemble of target/(receptor), in this case the SARSCoV-2 S-protein:ACE2 interface, conformations are generated for use in molecular docking to implicitly account for the conformational flexibility of the target and its presumed ligand-binding site $^{12-13}$. Typically the ensemble of target structures (conformations) are generated through the use of standard all-atom molecular dynamics (MD) simulations and structural clustering ${ }^{12-13}$. By accounting for the conformational diversity of the receptor ensemble docking enhances the likelihood of identifying predicted hits (enrichment), which may be lost when screening against a single conformation of the target ${ }^{12}$. We have previously applied this technique to derive experimentally-verified hits for several protein targets to treat diseases ranging from bacterial infections to osteoporosis ${ }^{14-22}$. For this work three phases of calculations were performed: structural modeling, molecular simulations (ensemble building), and small-molecule docking (in silico ligand screening)

\section{$\underline{\text { Structural Modeling and Molecular Dynamics Preparation }}$}

Recent structural modeling work by $\mathrm{Xu}$ et al. suggested that the S-protein from SARS-CoV-2has a strong binding affinity with the ACE2 receptor ${ }^{2}$. However, the coordinates of the structural model were not provided. Therefore, we generated a model of the SARS-CoV-2 S-protein-ACE2 complex (provided with the supplementary material). Using SWISS-MODEL ${ }^{23-24}$, the sequences for the COVID-19 S-protein (NCBI Reference Sequence: YP_009724390.1) and ACE2 receptor were used along with the crystal structure of the SARS-CoV S-protein in complex with ACE2 (PDB: 2AJF), as a template, to generate a model of the SARS-CoV-2 S-protein and ACE2 receptor complex. A rendering of the complex is provided in Figure 1. 
To prepare the system for molecular dynamics simulations, the structural model was centered in a periodic simulation box of $9.895 \mathrm{~nm} \times 8.525 \mathrm{~nm} \times 17.415 \mathrm{~nm}$ and solvated in the (CHARMM) TIP3P water model ${ }^{25}$ using the gmx editconf and gmx solvate tools from the GROMACS molecular dynamics simulation suite ${ }^{26}$. As the total charge of the S-protein-receptor complex was non-zero, 21 sodium ions were added to neutralize the system. To remove potential clashes between the solvent, ions, and S-protein-receptor complex, energy minimization calculations (using GROMACS with the CHARMM36 force-field ${ }^{27}$ ) were performed with a convergence criterion of $1000 \mathrm{~kJ} / \mathrm{mol} \mathrm{nm}^{-1}$. Following the energy minimization, a short, $1 \mathrm{~ns}$ NPT relaxation simulation was performed to relax the box dimensions. For the NPT simulation, the pressure and temperature were controlled using the Berendsen baro/thermostat ${ }^{28}$, and the simulation was performed using an integration time-step of $2 \mathrm{fs}$. For both NPT relaxation simulation and energy minimization calculations, short-range interactions were treated with a smooth force-switch cutoff of $1.2 \mathrm{~nm}$, and long-range electrostatics were treated using the PME (Particle-Mesh-Ewald) formalism, as implemented within GROMACS ${ }^{29}$. For the relaxation simulation, hydrogen-bonds were restrained with the LINCS algorithm ${ }^{30-31}$.

\section{$\underline{\text { Restrained Temperature Replica-Exchange Molecular Dynamics }}$}

The critical requirement of ensemble docking is the generation of an ensemble that captures a wide array of the conformational space of the target receptor. As we are most interested in the virus-host protein-protein interface (and subsequently the viral S-protein's host receptor recognition domain), we performed a restrained temperature replica-exchange molecular dynamics simulation (T-REMD), with 46 replicates at temperatures from $310 \mathrm{~K}$ to $350 \mathrm{~K}$, of the S-protein:ACE2 receptor complex with residues more than $1.5 \mathrm{~nm}$ from the protein-protein interface held fixed by harmonic restraints (with force-constants of $1000 \mathrm{~kJ} / \mathrm{mol} \mathrm{nm}^{-1}$ ). The T- 
REMD simulations generated trajectories that were $40 \mathrm{~ns}$ in length and the last $35 \mathrm{~ns}$ of each simulation used for analysis ( $35 \mathrm{~ns}$ per replicate for a total of $1.61 \mu \mathrm{s}$ of sampling). Simulations were performed using the GROMACS simulation suite (as compiled on the ORNL SUMMIT supercomputer) within the NPT ensemble with an integration time-step of $2 \mathrm{fs}$, a frame-saving rate of 10ps, and with exchange attempts every 500ps (with an exchange rate of $\sim 20 \%$ obtained throughout the simulation). Short-range interactions and long-range electrostatics were computed using the same cutoffs as those used in the NPT relaxation simulations (see Structural Modeling and Molecular Dynamics Preparation). The temperature was maintained with the V-rescale thermostat ${ }^{32}$, while the pressure was maintained using the Berendsen barostat ${ }^{28}$. The Berendsen barostat was used to accommodate the use of position restraints within the simulation. As with the NPT relaxation, all hydrogen-bonds were subjected to constraints, using the LINCS algorithm ${ }^{30-31}$.

Following the T-REMD simulation, frames from all replicates (regardless of temperature) were concatenated into a single file and used for structural clustering. Root-mean-squared (RMSD) based structural clustering of the conformations defined by the heavy-atoms of each side-chain within the S-protein:ACE2 receptor complex was performed with the gmx cluster tool of GROMACS using the GROMOS clustering algorithim ${ }^{33}$ and a cutoff of $0.2 \mathrm{~nm}$. Six clusters were identified via this clustering method and are provided in the Supplementary Material (SI) for interested readers.

After identifying the six clusters noted above, the interface region (see figure 1) of each structure was extracted and converted, using Autodock Tools ${ }^{34}$, into PDBQT formatted files for subsequent molecular docking calculations.

\section{Ligand Library Choice}


As the aim of this work was to identify previously approved small-molecules (along with metabolites, illicit drug compounds, and natural products) for repurposing in treating COVID-19 infections, we made use of the SWEETLEAD molecular library ${ }^{35}$. Each ligand (small-molecule) within the SWEETLEAD library was converted from SDF format to the PDBQT format used in Autodock Vina (Vina) by first converting the SDF format to mol2 using MOE2016 ${ }^{36}$ and then Autodock tools ${ }^{34}$. It is important to note that the library itself contains multiple "copies" of ligand entries with the same name; however, these repeat entries are isomers of the ligands within the library. For completeness, all isomers are considered as independent ligands and are also used in the docking calculations.

\section{$\underline{\text { in Silico Screening/Molecular Docking }}$}

Molecular docking calculations were performed using a unique POWER9 build of Autodock Vina $^{37}$ for SUMMIT. Two sets of docking calculations were performed, one targeting the Sprotein:ACE2 receptor interface (with the aim of identifying small-molecules for interface disruption) and the second calculation focusing on preventing S-protein recognition by binding to the ACE2 recognition region of the isolated S-protein. For both calculations, the same structural clusters identified from the MD simulations were used; however, in the case of the isolated S-protein, atoms belonging to the ACE2 receptor were removed. Docking calculations made use of a search box of $1.2 \mathrm{~nm} \times 1.2 \mathrm{~nm} \times 1 . \mathrm{x} 2 \mathrm{~nm}$, which was centered for, both the isolated S-protein and S-protein-ACE2 receptor interface, at the coordinates $(0,-2,-0.5)$ which roughly correspond to the location of the geometric center of the S-protein-ACE2-receptor interface. For all docking calculations, an exhaustiveness setting of 250 was used. To allow for the rapid evaluation of the SWEETLEAD library, while also making use of high search exhaustiveness, 
docking calculations were performed in parallel using a generic script-launching MPI wrapper compatible with SUMMIT (provided in the SI for interested readers).

Of the 9127 ligands within the SWEETLEAD library, only a subset could be successfully scored by Vina due to the imposed search box restriction and limits on rotatable bonds (see Tables 1 and 2). As a practical matter, we focus the discussion of our results on ligands with Vina scores better than $-7 \mathrm{kcal} / \mathrm{mol}$ (corresponding to the top $\sim 0.09 \%$ Vina scores) for the protein-protein interface target and $-6.2 \mathrm{kcal} / \mathrm{mol}$ (also corresponding to $\sim 0.09 \%$ of Vina scores) for the isolated S-protein target. After scoring each of the ligand-protein interface complex geometries generated during docking, the top four ligand-interface complexes were extracted for additional analysis using $\mathrm{MOE}^{36}$. For the top four ligand-interface complexes, energy minimization was performed in MOE with default convergence setting and with the MMF94x force-field ${ }^{38}$ to refine the ligand-interface. The refined structures were then used to generate 2D interaction maps between the ligand and the protein residues of the S-protein-ACE2 receptor interface and the isolated S-protein.

Table 1 Number of successful docking calculations for each cluster (receptor conformation) for the combined Sprotein-ACE2 interface target. The total number of successful docking calculations are also noted.

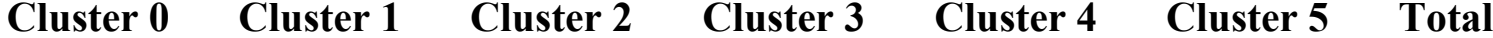

8574

8587

8581

8578

8621

8634

51575

Table 2. Number of successful docking calculations for each cluster (receptor conformation) for the Isolated Sprotein target. The total number of successful docking calculations are also noted.
Cluster 0
Cluster 1
Cluster 2
Cluster 3
Cluster 4
Cluster 5 Total

8483

8510

8526

8561

8523

8214

50817

\section{Results \& Discussion}


Ensemble docking produces multiple scores and poses for each ligand-target conformation. As noted in tables 1 and 2 (see Methods), over 100,000 docking calculations were performed with 51,575 ligand-target scores obtained for the S-protein:ACE2 receptor interface and 50,817 ligand-target scores for the ACE2 recognition region of the isolated S-protein. For ease of use for future studies we provide complete tables of the top pose scores for the interface and isolated targets in the SI, with 8669 scores provided for ligands binding to the S-protein:ACE2 receptor interface system and 8589 scores for ligands binding to the isolated S-protein's ACE2 recognition domain. Below we highlight seven ligands within the top $\sim 0.09 \%$ of all scores, for each target, respectively, that we believe may be of particular interest for experimental evaluation.

\section{Docking Results for the Host-Virus Interface (S-protein-ACE2 Receptor Complex)}

Of the 51575 docking calculations performed, 47 S-protein:ACE2 interface-ligand complexes were identified with scores equal to or better than the score threshold, and of the poses identified, 41 have ligands that are reported (as noted in the ZINC15 database $^{39}$ ) to be available for purchase, with 21 denoted as having regulatory approvals (Table 3).

Table 3. Top scoring ligands for S-protein:ACE2 receptor interface that have undergone regulatory review in the USA or elsewhere (as annotated in the ZINC15 database). A complete ranking is provided in the SI

\begin{tabular}{l|rl}
\hline Name (Obtained from the SWEETLEAD) & \multicolumn{1}{l}{ Vina } \\
\hline pemirolast & -7.4 & $\underline{\text { ZINC5783214 }}$ \\
\hline benserazide & -7.4 & $\underline{\text { ZINC3830273 }}$ \\
\hline Natural Product: luteolin-monoarabinoside & -7.4 & $\underline{\text { ZINC18185774 }}$ \\
\hline pyruvic acid calcium isoniazid & -7.3 & $\underline{\text { ZINC4974291 }}$ \\
\hline Natural Product: quercetol;quercitin & -7.3 & $\underline{\text { ZINC3869685 }}$ \\
\hline protirelin & -7.3 & $\underline{\text { ZINC4096261 }}$ \\
\hline carbazochrome & -7.2 & $\underline{\text { ZINC100029428 }}$ \\
\hline nitrofurantoin & -7.2 & $\underline{\text { ZINC3875368 }}$ \\
\hline benserazide & -7.2 & $\underline{\text { ZINC3830273 }}$ \\
\hline carbazochrome & -7.1 & $\underline{\text { ZINC100045148 }}$ \\
\hline
\end{tabular}




\begin{tabular}{lrl} 
sapropterin & -7.1 & ZINC13585233 \\
\hline Vidarabine & -7.1 & $\underline{\text { ZINC970363 }}$ \\
\hline Natural Product: eriodictyol & -7.1 & $\underline{\text { ZINC58117 }}$ \\
\hline tazobactum & -7.1 & $\underline{\text { ZINC3787060 }}$ \\
phenformin hcl & -7 & $\underline{\text { ZINC5851063 }}$ \\
carbazochrome & -7 & $\underline{\text { ZINC100045148 }}$ \\
\hline carbazochrome & -7 & $\underline{\text { ZINC100045148 }}$ \\
\hline vildagliptin & -7 & $\underline{\text { ZINC100003507 }}$ \\
\hline Natural product: demethyl-coclaurine & -7 & $\underline{\text { IINC896041 }}$ \\
\hline
\end{tabular}

The top pose-scores for each ligand are provided in the SI. Of the top 41 ranked compounds, we selected four (with scores ranging from -7.4 to -7.1) to highlight based on their poses, which are represented in figure 2. The highlighted compounds from our screening are: pemirolast $^{40-41}$ (ZincID: 5783214), isoniazid pyruvate (ZincID: 4974291), nitrofurantoin (ZincID: 3875368), and eriodictyol (ZincID: 58117). Of the four small-molecules shown in figure 2, the top-ranked, pemirolast, is an anti-allergy medication or for use in treating chronic asthma $^{40-41}$, while the second and third of the highlighted hits are related to well-known antibiotics, with nitrofurantoin an antibiotic for use against urinary tract infections ${ }^{42}$ and isoniazid pyruvate being a metabolite of the tuberculous antibiotic Isoniazid ${ }^{43}$. The last, Eriodictyol, is a flavanone found in Herba Santa and is a traditional herbal remedy used for asthma and treating colds ${ }^{44}$.

Figures (3-6) provide a detailed two-dimensional accounting of the ligand-interface interactions and may be of interest to researchers interested in identifying novel small-molecule therapeutics. These interaction maps indicate that three of the four top hits (isoniazid pyruvate, nitrofurantoin, and eriodictyol) each contain twice as many interactions with the ACE2 receptor than the S-protein, while pemirolast has equal amounts of interactions between the two proteins. Further, the second through fourth top hits had 3 to 6 more ligand-residue interactions. As the 
binding of the S-protein to ACE2 is undesirable, it is preferable to diminish ligand-interface interactions that may bridge, and therefore stabilize, the interaction between the S-protein and the ACE2 receptor. While the top hit, pemirolast, has the strongest binding affinity, initial repurposing may be better suited to isoniazid pyruvate, nitrofurantoin, or eriodictyol.

\section{Docking Results for the Isolated Virus S-protein Host Recognition Domain}

As noted in the previous section, docking to the interface may unintentionally identify interface stabilizing compounds (which cannot be easily identified a priori). To avoid this potential pitfall, we also performed docking calculations targeting the receptor recognition region of the S-protein itself. As the target is no longer a buried pocket (as was the case for the interface), we expect that therapeutic compounds that have not been optimized for the 'shallow' protein surface will have weaker binding affinities than the interface and as such, we adjusted the criteria for the selection of ligands for discussion to be those that have Vina scores better than $-6.2 \mathrm{kcal} / \mathrm{mol}$. Using the new score threshold, a total of 30 ligand-receptor poses were obtained (see SI for complete ranking); however, of these 30 only 3 were annotated in the ZINC15 database to have regulatory data from the US FDA or similar agencies in other countries. The three top-scoring complexes were obtained using the ligands: Cepharanthine, Ergoloid, and Hypericin. Cepharanthine and Hypericin are both natural products with both having been the subject of multiple studies on their ability to act as antiviral ${ }^{45-48}$ agents (including against coronaviruses ${ }^{46,48}$ ), while Ergoloid is an FDA approved ${ }^{49}$ drug component of interest in dementia therapies ${ }^{50-52}$. The docking poses for each of these compounds are presented in figure 7 and the associated interaction networks between these ligands and the isolated S-protein presented in figures 8-10. The renderings of the poses and interaction networks show favorable ring-protein interactions which may be driving the ligand-binding interactions. 


\section{Conclusion}

Prior work has demonstrated that the COVID-19 associated SARS-CoV-2 virus shares the ACE2 receptor as an entry point for infection with the SARS-CoV. Here we made use of enhanced sampling molecular simulations of currently available structure models of the S-protein of SARS-CoV-2 binding with the ACE2 receptor to generate an ensemble of configurations for ensemble docking. Further, we have made use of this ensemble to screen the SWEETLEAD library against the interface and isolated viral S-protein. Our docking calculations have identified 47 potential hits for the interface, with 21 having regulatory data and 20 of these being available for purchase, and 30 for the S-protein alone, with 3 top hits having ZINC15 annotations indicating regulatory data existing.

With regards to our interface docking results, we highlighted seven of our top compounds, namely those that have been previously used as drugs or as potential drugs. Of the highlight interface-binding compounds, three (nitrofurantoin, isoniazid pyruvate, and eriodictyol) were found to have a preference for residues belonging to the ACE2 receptor portion of the interface, and we hypothesize that these favorable interactions may in-turn limit the binding of the SARS-CoV-2 S-protein with the ACE2 receptor, thus restricting infection. With regards to screening against the isolated S-protein's receptor recognition region, the three identified compounds of interest were: Cepharanthine, Ergoloid, and Hypericin, with Cepharanthine and Hypericin having prior data suggesting their use as anti-viral agents against other coronaviruses $^{46,48}$. Given the results from both sets of docking calculations, our work suggests that at least the seven compounds identified here would be reasonable initial compounds for experimental investigations in limiting SARS-CoV-2's virus-host interactions. Furthermore, by providing an extensive ensemble-docking generated ranking of small-molecules, we provide an 
important filtering for future experimental studies targeting the infection pathway of SARSCoV-2.

\section{Supplementary Information}

We provide for the interested reader a substantial portion of our raw data, including the PDB structures of the complex generated from SWISSMODEL, excel spreadsheets containing the best-pose Vina scores for each ligand along with internal ligand ID. The excel spreadsheets also contain the ZINC-ID and the commercial availability of the top 47 ligands identified by our interface docking calculations and the top 30 ligands identified by the isolated S-protein docking calculations. In addition to our docking results, MD input files and coordinate files for the MD derived structural clusters are also provided. To facilitate others in performing similar docking calculations, the $\mathrm{C}$ code and accompanying bash-scripts used in this study to execute Vina over an arbitrary number of SUMMIT nodes are also provided.

\section{Acknowledgments}

We acknowledge support from the Oak Ridge Leadership Computing Facility.

This manuscript has been authored by UT-Battelle, LLC under Contract No. DE-AC05-000R22725 with the U.S. Department of Energy. The United States Government retains and the publisher, by accepting the article for publication, acknowledges that the United States Government retains a non-exclusive, paid-up, irrevocable, world-wide license to publish or reproduce the published form of this manuscript, or allow others to do so, for United States Government purposes. The Department of Energy will provide public access to these results of federally sponsored research in accordance with the DOE Public Access Plan (http://energy.gov/downloads/doe-public-access-plan ).

\section{References}

1. Li, Q.-H.; Ma, Y.-H.; Wang, N.; Hu, Y.; Liu, Z.-Z., New Coronavirus-Infected Pneumonia Engulfs Wuhan. Asian Toxicology Research 2020, 1.

2. $\quad \mathrm{Xu}, \mathrm{X}$.; Chen, P.; Wang, J.; Feng, J.; Zhou, H.; Li, X.; Zhong, W.; Hao, P., Evolution of the novel coronavirus from the ongoing Wuhan outbreak and modeling of its spike protein for risk of human transmission. Science China Life Sciences 2020, 1-4. 
3. Guarner, J., Three Emerging Coronaviruses in Two DecadesThe Story of SARS, MERS, and Now COVID-19. American Journal of Clinical Pathology 2020.

4. Zhang, Y., Initial genome release of novel coronavirus. 2020.

5. Prabakaran, P.; Xiao, X.; Dimitrov, D. S., A model of the ACE2 structure and function as a SARSCoV receptor. Biochem Bioph Res Co 2004, 314 (1), 235-241.

6. $\quad$ Li, W.; Zhang, C.; Sui, J.; Kuhn, J. H.; Moore, M. J.; Luo, S.; Wong, S. K.; Huang, I. C.; Xu, K.; Vasilieva, N., Receptor and viral determinants of SARS-coronavirus adaptation to human ACE2. The EMBO journal 2005, 24 (8), 1634-1643.

7. Lu, R.; Zhao, X.; Li, J.; Niu, P.; Yang, B.; Wu, H.; Wang, W.; Song, H.; Huang, B.; Zhu, N., Genomic characterisation and epidemiology of 2019 novel coronavirus: implications for virus origins and receptor binding. The Lancet 2020.

8. Wan, Y.; Shang, J.; Graham, R.; Baric, R. S.; Li, F., Receptor recognition by novel coronavirus from Wuhan: An analysis based on decade-long structural studies of SARS. Journal of Virology 2020.

9. Hoffmann, M.; Kleine-Weber, H.; Krüger, N.; Müller, M.; Drosten, C.; Pöhlmann, S., The novel coronavirus 2019 (2019-nCoV) uses the SARS-coronavirus receptor ACE2 and the cellular protease TMPRSS2 for entry into target cells. bioRxiv 2020, 2020.01.31.929042.

10. Van Norman, G. A., Drugs, devices, and the FDA: Part 1: an overview of approval processes for drugs. JACC: Basic to Translational Science 2016, 1 (3), 170-179.

11. Wang, C.; Horby, P. W.; Hayden, F. G.; Gao, G. F., A novel coronavirus outbreak of global health concern. The Lancet 2020.

12. Amaro, R. E.; Baudry, J.; Chodera, J.; Demir, Ö.; McCammon, J. A.; Miao, Y.; Smith, J. C., Ensemble docking in drug discovery. Biophys J 2018, 114 (10), 2271-2278.

13. Huang, S. Y.; Zou, X., Ensemble docking of multiple protein structures: considering protein structural variations in molecular docking. Proteins: Structure, Function, and Bioinformatics 2007, 66 (2), 399-421.

14. Pi, M.; Kapoor, K.; Wu, Y.; Ye, R.; Senogles, S. E.; Nishimoto, S. K.; Hwang, D.-J.; Miller, D. D.; Narayanan, R.; Smith, J. C., Structural and functional evidence for testosterone activation of GPRC6A in peripheral tissues. Mol Endocrinol 2015, 29 (12), 1759-1773.

15. Pi, M.; Kapoor, K.; Ye, R.; Nishimoto, S. K.; Smith, J. C.; Baudry, J.; Quarles, L. D., Evidence for osteocalcin binding and activation of GPRC6A in $\beta$-cells. Endocrinology 2016, 157 (5), 1866-1880.

16. Evangelista, W.; Weir, R. L.; Ellingson, S. R.; Harris, J. B.; Kapoor, K.; Smith, J. C.; Baudry, J., Ensemble-based docking: From hit discovery to metabolism and toxicity predictions. Bioorgan Med Chem 2016, 24 (20), 4928-4935.

17. Abdali, N.; Parks, J. M.; Haynes, K. M.; Chaney, J. L.; Green, A. T.; Wolloscheck, D.; Walker, J. K.; Rybenkov, V. V.; Baudry, J.; Smith, J. C., Reviving antibiotics: efflux pump inhibitors that interact with AcrA, a membrane fusion protein of the AcrAB-TolC multidrug efflux pump. ACS infectious diseases 2017, 3 (1), 89-98.

18. Haynes, K. M.; Abdali, N.; Jhawar, V.; Zgurskaya, H. I.; Parks, J. M.; Green, A. T.; Baudry, J.; Rybenkov, V. V.; Smith, J. C.; Walker, J. K., Identification and Structure-Activity Relationships of Novel Compounds that Potentiate the Activities of Antibiotics in Escherichia coli. Journal of medicinal chemistry 2017, 60 (14), 6205-6219.

19. Velazquez, H. A.; Riccardi, D.; Xiao, Z.; Quarles, L. D.; Yates, C. R.; Baudry, J.; Smith, J. C., Ensemble docking to difficult targets in early-stage drug discovery: Methodology and application to fibroblast growth factor 23. Chemical biology \& drug design 2018, 91 (2), 491-504.

20. Darzynkiewicz, Z. M.; Green, A. T.; Abdali, N.; Hazel, A.; Fulton, R. L.; Kimball, J.; Gryczynski, Z.; Gumbart, J. C.; Parks, J. M.; Smith, J. C., Identification of binding sites for efflux pump inhibitors of the AcrAB-TolC component AcrA. Biophys J 2019, 116 (4), 648-658. 
21. Pi, M.; Kapoor, K.; Ye, R.; Smith, J. C.; Baudry, J.; Quarles, L. D., GPCR6A is a molecular target for the natural products gallate and EGCG in green tea. Molecular nutrition \& food research 2018, 62 (8), 1700770.

22. Xiao, Z.; Baudry, J.; Cao, L.; Huang, J.; Chen, H.; Yates, C. R.; Li, W.; Dong, B.; Waters, C. M.; Smith, J. C., Polycystin-1 interacts with TAZ to stimulate osteoblastogenesis and inhibit adipogenesis. The Journal of clinical investigation 2018, 128 (1), 157-174.

23. Biasini, M.; Bienert, S.; Waterhouse, A.; Arnold, K.; Studer, G.; Schmidt, T.; Kiefer, F.; Cassarino, T. G.; Bertoni, M.; Bordoli, L., SWISS-MODEL: modelling protein tertiary and quaternary structure using evolutionary information. Nucleic Acids Res 2014, 42 (W1), W252-W258.

24. Schwede, T.; Kopp, J.; Guex, N.; Peitsch, M. C., SWISS-MODEL: an automated protein homologymodeling server. Nucleic Acids Res 2003, 31 (13), 3381-3385.

25. Bjelkmar, P.; Larsson, P.; Cuendet, M. A.; Hess, B.; Lindahl, E., Implementation of the CHARMM Force Field in GROMACS: Analysis of Protein Stability Effects from Correction Maps, Virtual Interaction Sites, and Water Models. J Chem Theory Comput 2010, 6 (2), 459-466.

26. Van der Spoel, D.; Lindahl, E.; Hess, B.; Groenhof, G.; Mark, A. E.; Berendsen, H. J. C., GROMACS: Fast, flexible, and free. J Comput Chem 2005, 26 (16), 1701-1718.

27. Huang, J.; MacKerell Jr, A. D., CHARMM36 all-atom additive protein force field: Validation based on comparison to NMR data. J Comput Chem 2013, 34 (25), 2135-2145.

28. Berendsen, H. J. C.; Postma, J. P. M.; Vangunsteren, W. F.; Dinola, A.; Haak, J. R., MolecularDynamics with Coupling to an External Bath. J Chem Phys 1984, 81 (8), 3684-3690.

29. Abraham, M. J.; Gready, J. E., Optimization of Parameters for Molecular Dynamics Simulation Using Smooth Particle-Mesh Ewald in GROMACS 4.5. J Comput Chem 2011, 32 (9), 2031-2040.

30. Hess, B.; Bekker, H.; Berendsen, H. J. C.; Fraaije, J. G. E. M., LINCS: A linear constraint solver for molecular simulations. J Comput Chem 1997, 18 (12), 1463-1472.

31. Hess, B., P-LINCS: A parallel linear constraint solver for molecular simulation. J Chem Theory Comput 2008, 4 (1), 116-122.

32. Bussi, G.; Donadio, D.; Parrinello, M., Canonical sampling through velocity rescaling. J Chem Phys 2007, $126(1)$.

33. Daura, X.; Gademann, K.; Jaun, B.; Seebach, D.; Van Gunsteren, W. F.; Mark, A. E., Peptide folding: when simulation meets experiment. Angewandte Chemie International Edition 1999, 38 (1-2), 236-240.

34. Huey, R.; Morris, G. M., Using AutoDock 4 with AutoDocktools: a tutorial. The Scripps Research Institute, USA 2008, 54-56.

35. Novick, P. A.; Ortiz, O. F.; Poelman, J.; Abdulhay, A. Y.; Pande, V. S., SWEETLEAD: an in silico database of approved drugs, regulated chemicals, and herbal isolates for computer-aided drug discovery. Plos One 2013, 8 (11).

36. Inc., C. C. G., Molecular operating environment (MOE). Chemical Computing Group Inc 1010 Sherbooke St. West, Suite\# 910, Montreal ...: 2016.

37. Trott, O.; Olson, A. J., AutoDock Vina: improving the speed and accuracy of docking with a new scoring function, efficient optimization, and multithreading. J Comput Chem 2010, 31 (2), 455-461.

38. Halgren, T. A., Merck molecular force field. I. Basis, form, scope, parameterization, and performance of MMFF94. J Comput Chem 1996, 17 (5-6), 490-519.

39. Sterling, T.; Irwin, J. J., ZINC 15-ligand discovery for everyone. J Chem Inf Model 2015, 55 (11), 2324-2337.

40. Kemp, J.; Bernstein, I.; Bierman, C.; Li, J.; Siegel, S.; Spangenberg, R.; Tinkelman, D., Pemirolast, a new oral nonbronchodilator drug for chronic asthma. Ann Allergy 1992, 68 (6), 488-491. 
41. Fujimiya, H.; Nakashima, S.; Miyata, H.; Nozawa, Y., Effect of a novel antiallergic drug, pemirolast, on activation of rat peritoneal mast cells: inhibition of exocytotic response and membrane phospholipid turnover. International Archives of Allergy and Immunology 1991, 96 (1), 62-67.

42. D'Arcy, P. F., Nitrofurantoin. Drug intelligence \& clinical pharmacy 1985, 19 (7-8), 540-547.

43. Timmins, G. S.; Deretic, V., Mechanisms of action of isoniazid. Molecular microbiology 2006, 62 (5), 1220-1227.

44. Ley, J. P.; Krammer, G.; Reinders, G.; Gatfield, I. L.; Bertram, H.-J., Evaluation of Bitter Masking Flavanones from Herba Santa (Eriodictyon californicum (H. \& A.) Torr., Hydrophyllaceae). J Agr Food Chem 2005, 53 (15), 6061-6066.

45. Gulick, R. M.; McAuliffe, V.; Holden-Wiltse, J.; Crumpacker, C.; Liebes, L.; Stein, D. S.; Meehan, P.; Hussey, S.; Forcht, J.; Valentine, F. T.; 150, f. t. A. C. T. G.; Teams, P., Phase I Studies of Hypericin, the Active Compound in St. John's Wort, as an Antiretroviral Agent in HIV-Infected Adults: AIDS Clinical Trials Group Protocols 150 and 258. Ann Intern Med 1999, 130 (6), 510-514.

46. Chen, H.; Muhammad, I.; Zhang, Y.; Ren, Y.; Zhang, R.; Huang, X.; Diao, L.; Liu, H.; Li, X.; Sun, X.; Abbas, G.; Li, G., Antiviral Activity Against Infectious Bronchitis Virus and Bioactive Components of Hypericum perforatum L. Front Pharmacol 2019, 10 (1272).

47. Shih, C.-M.; Wu, C.-H.; Wu, W.-J.; Hsiao, Y.-M.; Ko, J.-L., Hypericin inhibits hepatitis C virus replication via deacetylation and down-regulation of heme oxygenase-1. Phytomedicine 2018, 46, 193198.

48. Kim, D. E.; Min, J. S.; Jang, M. S.; Lee, J. Y.; Shin, Y. S.; Park, C. M.; Song, J. H.; Kim, H. R.; Kim, S.; Jin, Y.-H., Natural Bis-Benzylisoquinoline Alkaloids-Tetrandrine, Fangchinoline, and Cepharanthine, Inhibit Human Coronavirus OC43 Infection of MRC-5 Human Lung Cells. Biomolecules 2019, 9 (11), 696. 49. Food, U.; Administration, D., Drugs@ FDA: FDA approved drug products. 2018.

50. Bicalho, B.; Giolo, J. M.; Lilla, S.; De Nucci, G., Identification and human pharmacokinetics of dihydroergotoxine metabolites in man: preliminary results. Biopharmaceutics \& Drug Disposition 2008, 29 (1), 17-28.

51. Howland, R. H., Alternative drug therapies for dementia. Journal of psychosocial nursing and mental health services 2011, 49 (5), 17-20.

52. van Loveren-Huyben, C.; Engelaar, H.; Hermans, M.; Van der Bom, J.; Leering, C.; Munnichs, J., Double-blind Clinical and Psychologic Study of Ergoloid Mesylates (Hydergine ${ }^{\circledR}$ ) in Subjects with Senile Mental Deterioration. Journal of the American Geriatrics Society 1984, 32 (8), 584-588. 
Figures.

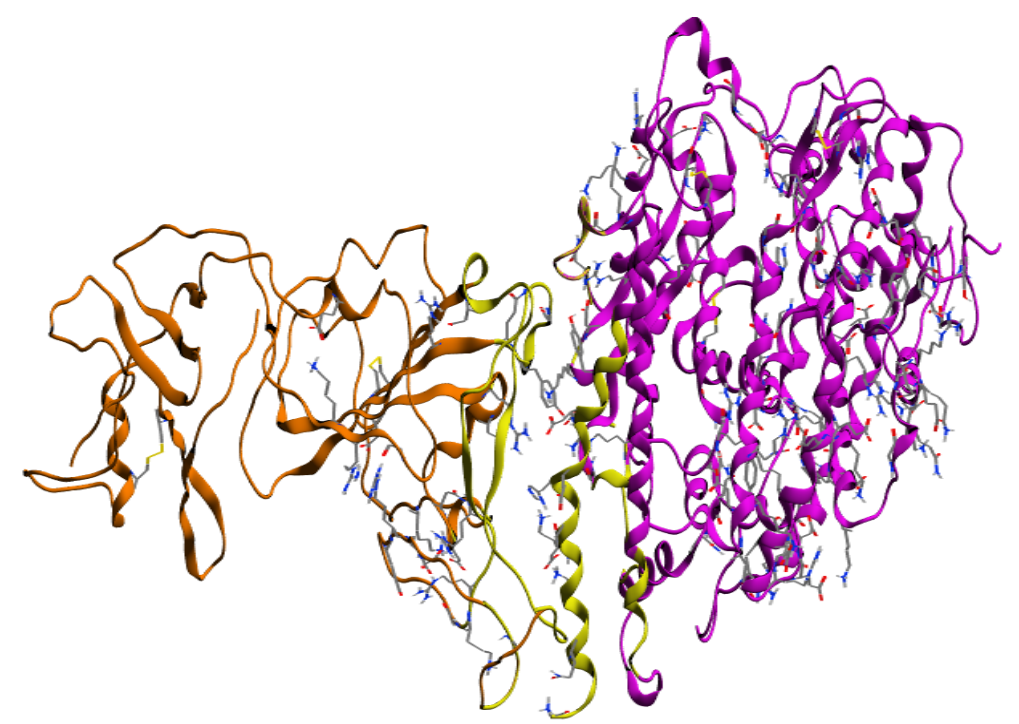

Figure 1) Rendering of nCoV-2019 S-protein and ACE2 receptor complex. Orange ribbons represent the S-protein, purple corresponds to ACE2, and yellow is a highlight of the interface targeted for docking. 


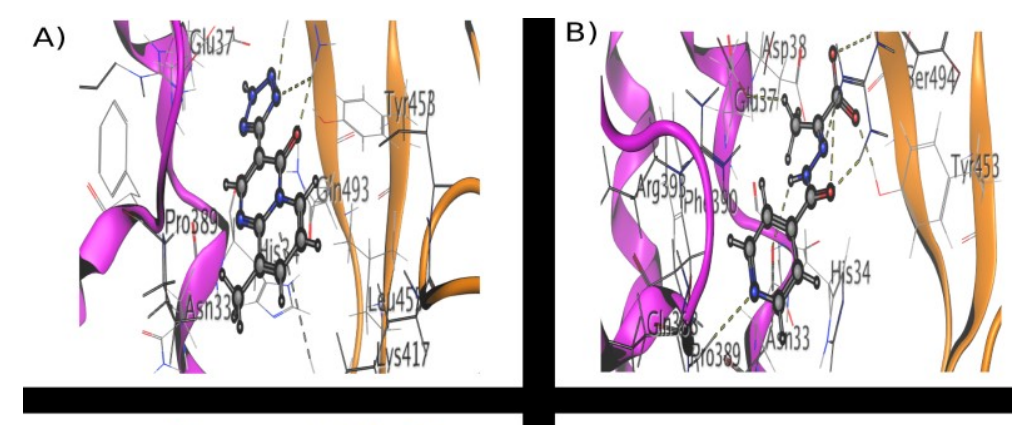

C)

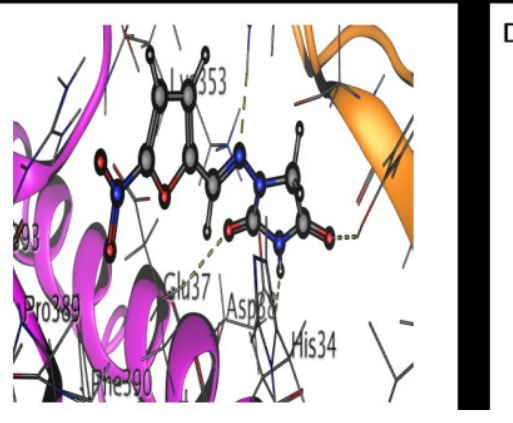

D)

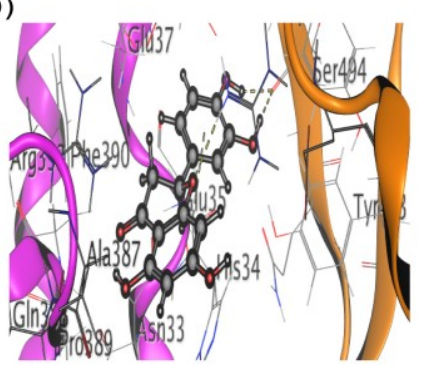

Figure 2) Renderings of four of the top scoring previously regulator approved small-molecules binding within the S-protein-ACE2 interface. A) pemirolast (ZincID: 5783214). B) isoniazid pyruvate (ZincID: 4974291). C) Nitrofurantoin (Zinc ID: 3875368). D) Eriodictyol (ZincID: 58117). Orange ribbons represent the S-protein and purple ribbons correspond to the ACE2 receptor 


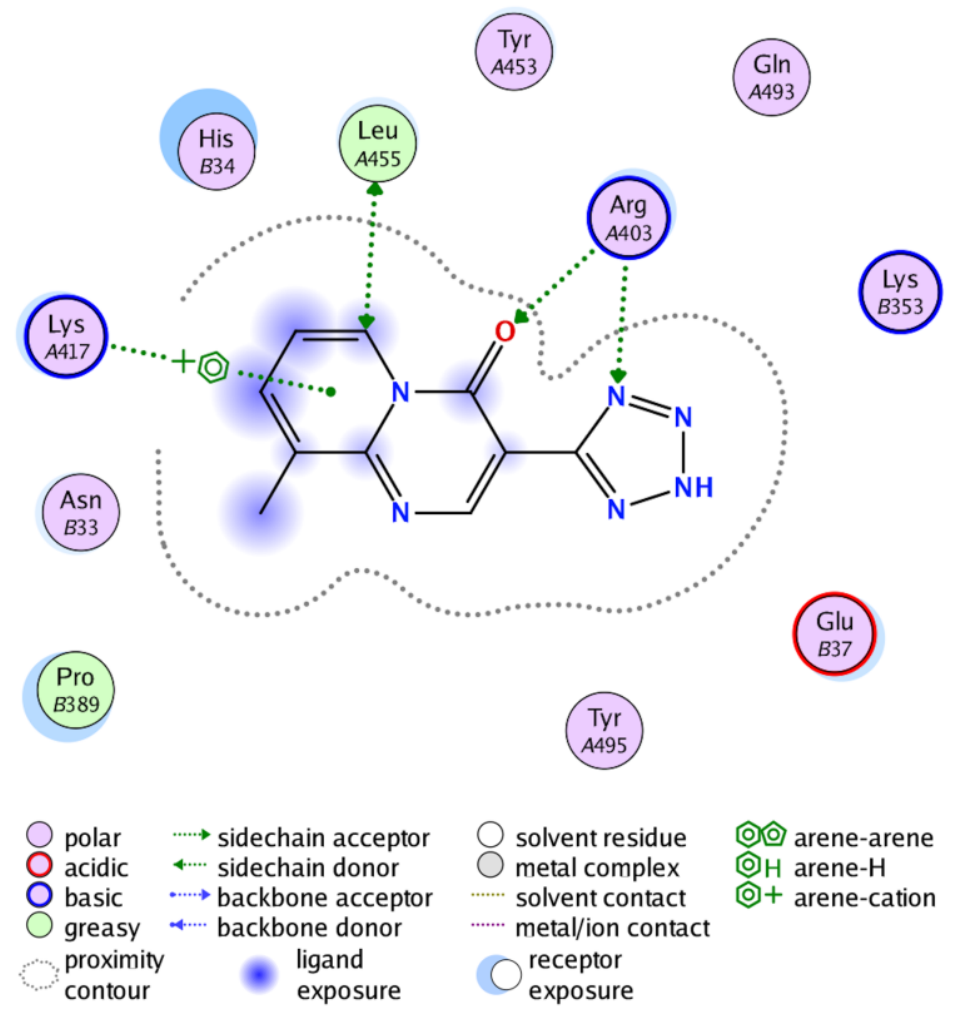

Figure 3) permirolast-protein Interface interaction diagram (generated from MOE2016). Residues with (A) correspond to the S-protein, while those with (B) correspond with the ACE2 receptor. 


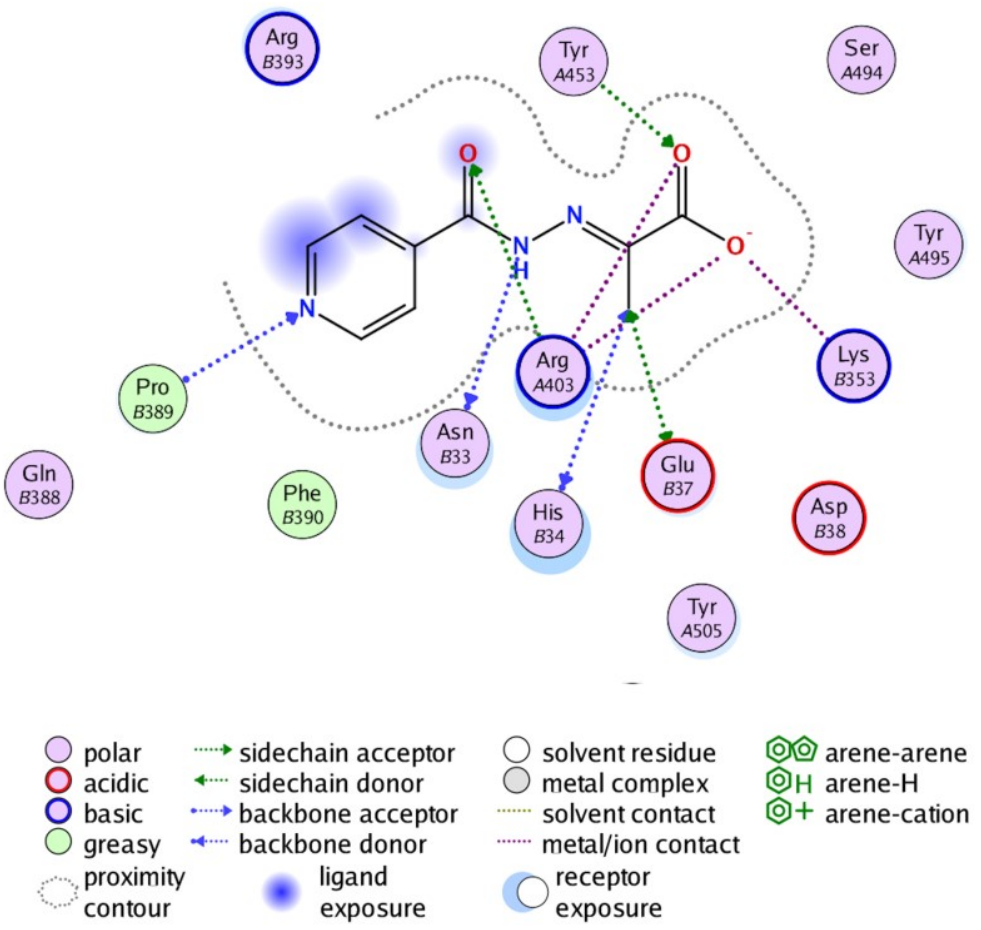

Figure 4) isoniazid pyruvate-protein Interface interaction diagram (generated from MOE2016). Residues with (A) correspond to the S-protein, while those with (B) correspond with the ACE2 receptor. 


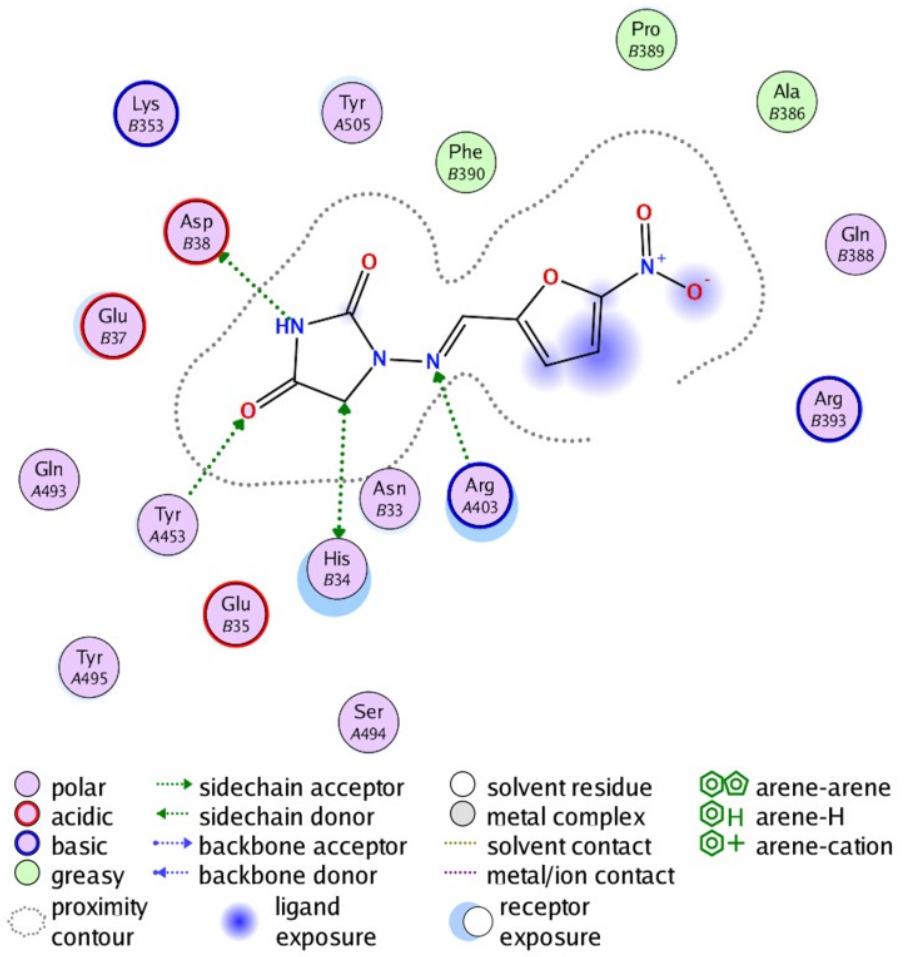

Figure 5) nitrofurantoin-protein Interface interaction diagram (generated from MOE2016). Residues with (A) correspond to the S-protein, while those with (B) correspond with the ACE2 receptor. 


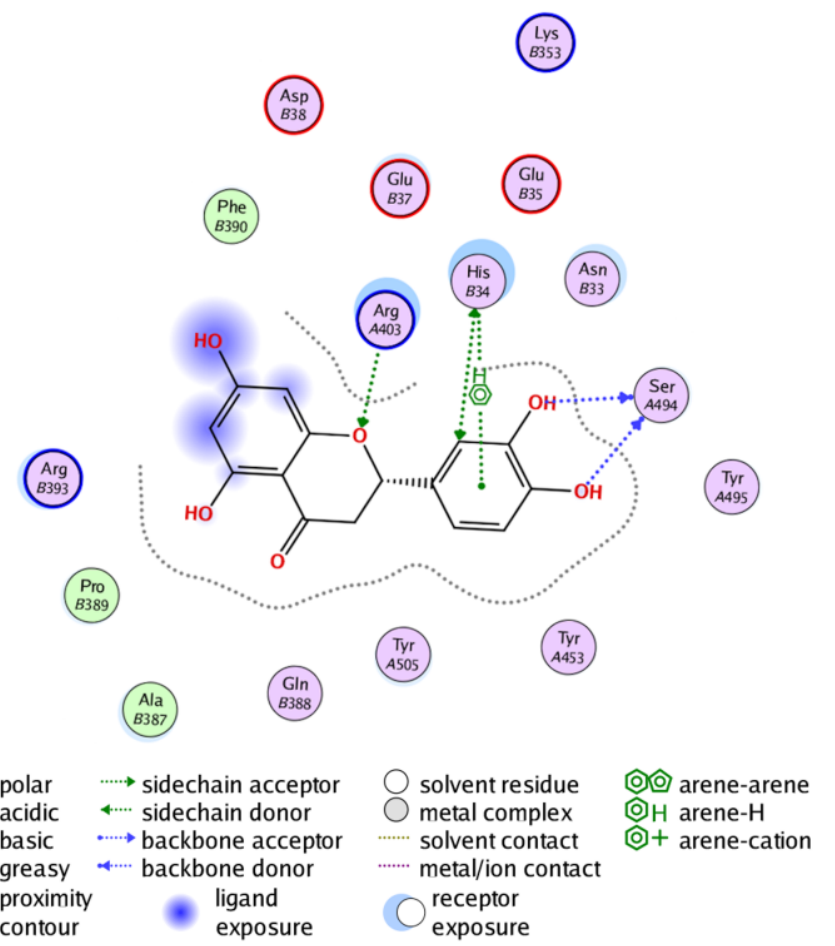

Figure 6) eriodictyol -protein Interface interaction diagram (generated from MOE2016). Residues with (A) correspond to the S-protein, while those with (B) correspond with the ACE2 receptor. 


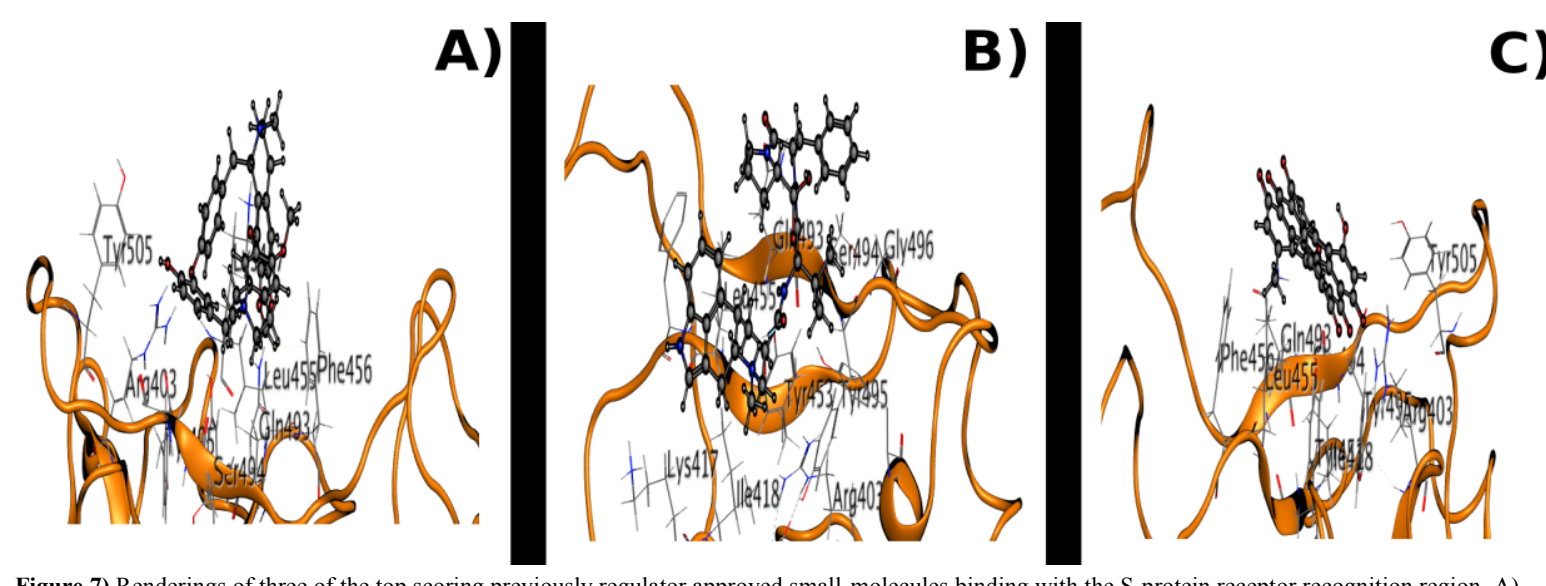

Figure 7) Renderings of three of the top scoring previously regulator approved small-molecules binding with the S-protein receptor recognition region. A) Cepharanthine (ZincID: 30726863). B) Ergoloid (ZincID: 3995616). C) Hypericin (Zinc ID: 3780340). Orange ribbons represent the S-protein. 


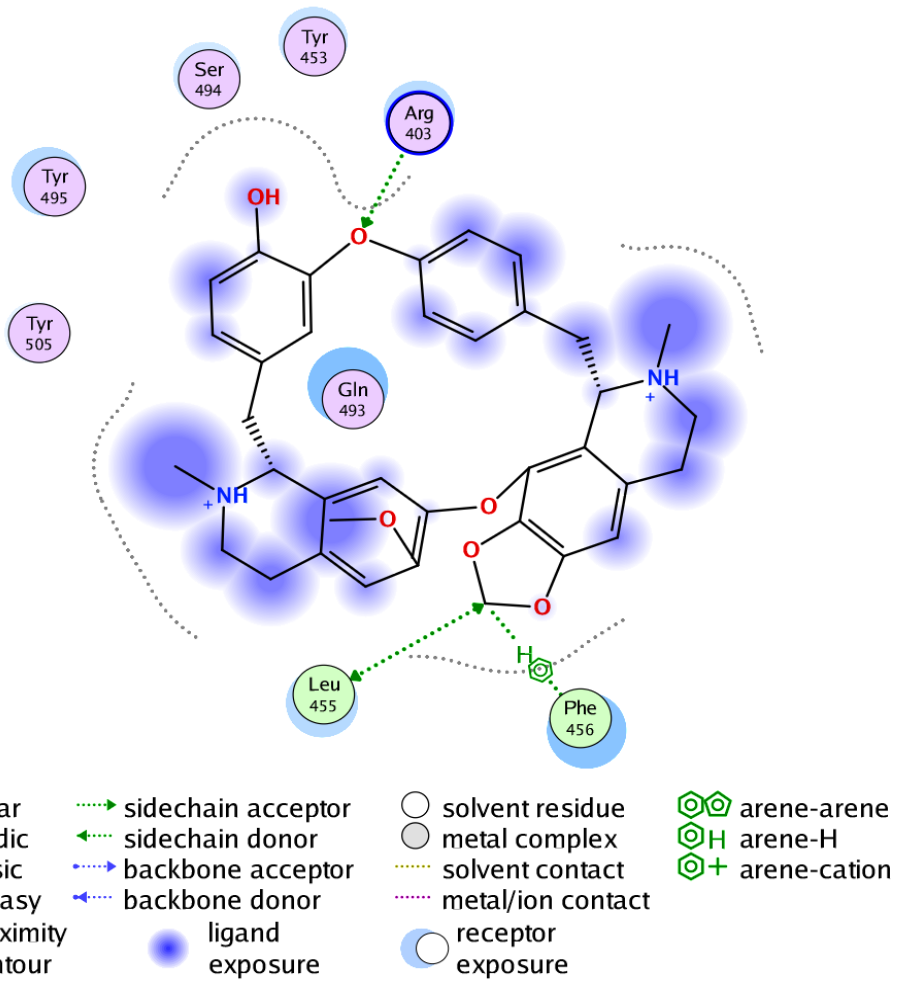

Figure 8) Cepharanthine-S-protein interaction diagram (generated from MOE2016). 


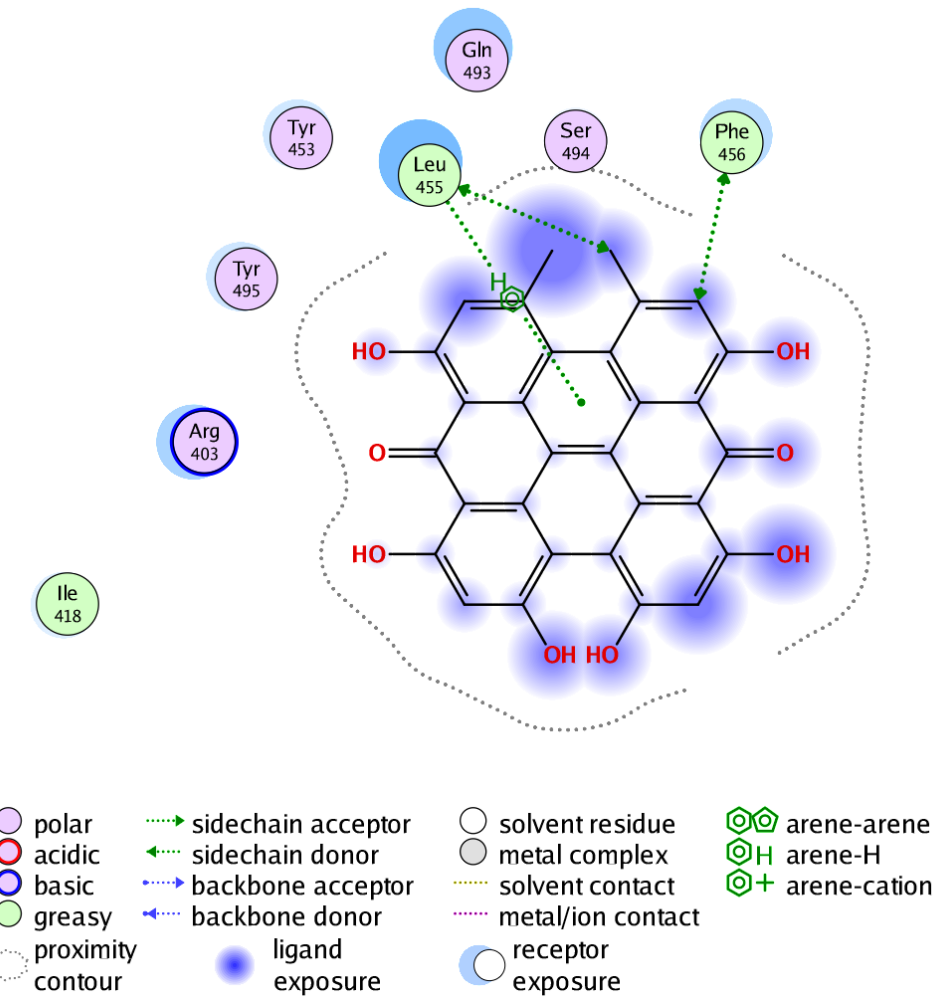

Figure 9) Hypericin-S-protein interaction diagram (generated from MOE2016). 


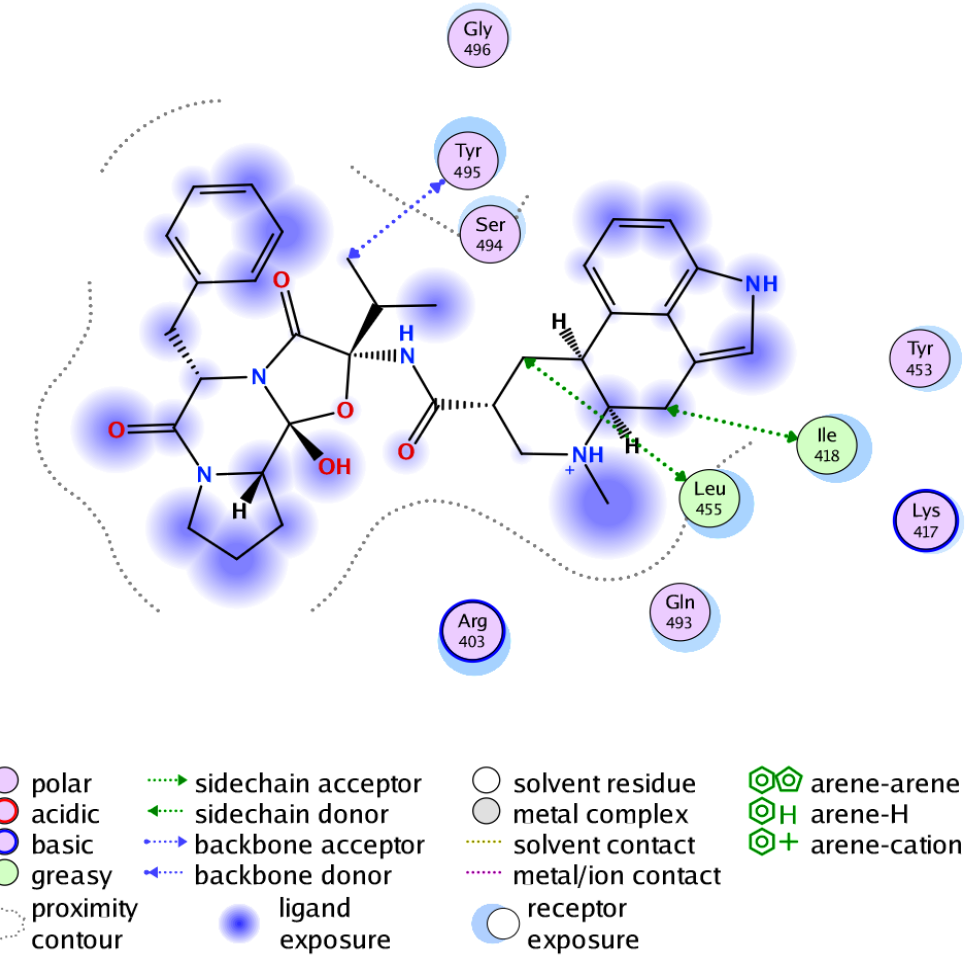

Figure 10) Ergoloid-S-protein interaction diagram (generated from MOE2016). 